\title{
Perampanel-Induced Cataplexy in a Young Male with Generalized Epilepsy
}

Kelsey Kenaan, ${ }^{1}$ Mohsin Zafar, MD, ${ }^{2}$ Ronnie Bond, MD, ${ }^{2}$ Barbara L. Gracious, MD ${ }^{2}$

\section{Abstract}

\section{Description}

Perampanel (Fycompa) is a newer anti-epileptic drug believed to exert its effects in the central nervous system by inhibiting post-synaptic glutamate receptors. However, the precise therapeutic mechanism is unknown. The most common neuropsychiatric side effect is affective dysregulation; there are also reports of psychosis. We describe a 32 year old African American male with recurring generalized tonic-clonic (GTC) seizures, who presented to our hospital with onset of mood lability for several months, after Perampanel was added to his antiepileptic medications. Perampanel administration was temporarily withheld, and subsequently on restarting, noted to be coincident with neuropsychiatric symptomatology, including motor weakness in emotional contexts. The mechanisms underlying cataplexy are complex and, in our patient, most likely induced by an interaction between Perampanel and the wakeful inhibition of the sublaterodorsal nucleus projections.

\section{Keywords}

cataplexy; narcolepsy; disorders of excessive somnolence; sleep wake disorders; perampanel; Fycompa; orexins; hypocretin; seizures; epilepsy; REM sleep behavior disorder; drug-related side effects and adverse reactions

\section{Introduction}

Perampanel (Fycompa) is an anticonvulsant agent approved by the US Food and Drug Administration (FDA) in 2012 as adjunctive therapy for the treatment of partial-onset seizures with or without secondary generalized seizures in patients aged 12 years or older. Perampanel is the first medication to be specifically designed with glutamate inhibition as its primary mechanism of action. ${ }^{2}$ The alleged mechanism of action involves non-competitive selective antagonism at the postsynaptic ionotropic alpha-amino-3-hydroxy-5-methyl-4-isoxazolepropionic acid (AMPA) receptor. By acting as a non-competitive inhibitor, perampanel allows glutamate to bind to its receptor, but inhibits the normal excitatory physiologic response. ${ }^{3}$

The most common side effects of perampanel include dizziness, somnolence, headache, fatigue, falls, ataxia, nausea, vertigo and back pain. ${ }^{1}$ Adverse psychiatric effects, including potential for affective changes, may possibly occur at a greater rate than some the older antiseizure agents. Phase III data documented irritability at dose-dependent rates of $3 \%$ with placebo, and $4 \%, 7 \%$ and $12 \%$ with 4,8 and 12 $\mathrm{mg} /$ day respectively. ${ }^{2}$ Per the FDA package insert, "in the controlled partial-onset seizure clinical trials, hostility- and aggression-related adverse reactions occurred in $12 \%$ and $20 \%$ of patients randomized to receive perampanel at doses of $8 \mathrm{mg}$ and $12 \mathrm{mg}$ per day, respectively, compared to $6 \%$ of patients in the placebo group." ${ }^{2}$ Homicidal ideation and/or threats were reported in $0.1 \%$ of the 4138 participants. ${ }^{1}$

We believe this case is the first reported of cataplexy related to perampanel use.

\section{Case Presentation}

A 32-year-old African American man with a history of HIV, generalized tonic-clonic seizure disorder, depression and anxiety presented to a

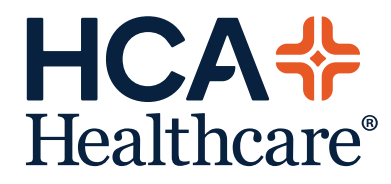

www.hcahealthcarejournal.com

(C) 2020 HCA Physician Services, Inc. d/b/a Emerald Medical Education
HCA Healthcare
Journal of Medicine 
hospital emergency department with worsening memory lapses and mood lability episodes. The most recent symptoms included bouts of laughter starting three days prior to presentation. He was taking levetiracetam $1000 \mathrm{mg}$, carbamazepine XR 400mg, and perampanel $10 \mathrm{mg}$, all once daily, for recurrent generalized tonic-clonic seizures. Durations of use were: levetiracetam, 2 years; carbamazepine, over 11 years; and perampanel, 7-8 months. The patient's symptoms began about 7-8 months before the ED presentation, starting with heightened fearfulness, followed by paranoia akin to PTSD, and then progressing to include memory loss and what was thought to be mood lability on evaluation in the emergency department. He denied previous similar symptoms, and there was no known family history of mental illness or narcolepsy per the family. His symptoms resulted in extreme social impairment; the patient had given up living independently and had moved back in with his parents so they could help care for him.

\section{Hospital Course}

The patient was admitted to the inpatient psychiatric unit for further evaluation and treatment of mood lability and abnormal behavior. As a probe into the etiology of his lability, it was noted during direct history and mental status examination that the lability was able to be manipulated by changing the topic from neutral to sad or humorous, and back to neutral, with congruent abrupt affect changes in the patient. Additionally, the patient's episodes of severe laughing or crying involved loss of muscle tone, resulting in his head initially falling to the side or front; he also fell to the floor several times. These findings were consistent with a clinical diagnosis of cataplexy. An EEG showed no presence of temporal lobe or other seizure activity, despite emotional changes precipitated by changes in conversational tone by the technician during the test.

Perampanel and carbamazepine were not given at admission, as the carbamazepine level was supratherapeutic and perampanel was non-formulary and not initially available. The cataplexy episodes resolved after 2 days, but on day 3 , the patient then suffered several seizures and was transferred to a medical unit. On hospital day 4 , he did not display cataplexy or lability of any kind. Per family's report, the patient had good seizure control on carbamazepine monotherapy. Poor compliance resulted in recurrent seizures and additional anticonvulsants added to his medications. The patient was restarted on all of his anti-epileptics with a plan to taper perampanel first if stable, followed by levetiracetam. Several hours after receiving a dose of perampanel, the patient became confused, and had visual hallucinations and severe agitation requiring physical restraints. New periods of emotional lability and muscle weakness precipitated by emotional contexts during social communication re-emerged, consistent with reduplicated cataplexy. Perampanel was again discontinued and the patient was observed for two additional days, during which time his memory improved, and he did not display any further cataplexy episodes, agitation, or endorse visual hallucinations.

\section{Discussion}

Cataplexy is defined as an emotionally triggered paralysis or sudden involuntary loss of muscle tone with preserved consciousness; it is a pathognomonic symptom of narcolepsy. ${ }^{4,5,6,7}$ Cataplexy presents alone, as well as comorbid with narcolepsy, Parkinson's disease, multiple sclerosis, head trauma, encephalitis and drop attack-types of seizures. ${ }^{6}$ Most cataplexy episodes are triggered by strong positive emotions such as laughter. Reported causes include drug-induced cataplexy, through the rapid withdrawal of tricyclic antidepressants ${ }^{6}$ or lamotrigine. ${ }^{8}$ The prevalence of isolated cataplexy is unknown, but the prevalence of cataplexy in those with excessive daytime somnolence has been reported as up to $29 \%{ }^{6}$

The differential diagnosis of cataplexy includes: migraine headaches, bradycardia with presyncope, delayed sleep-phase syndrome, temporal or partial complex/akinetic seizures, Niemann-Pick Type C disease, Coffin-Lowry syndrome, pontomedullary lesions, conversion disorder, malingering and chronic psychotic disorder. ${ }^{4,5}$

The exact mechanism behind cataplexy is still unknown, but it is hypothesized that orexin (also known as hypocretin), plays a major role. Orexin A and orexin B (also known as hypocretin 1 and hypocretin 2, respectively) are neuro- 


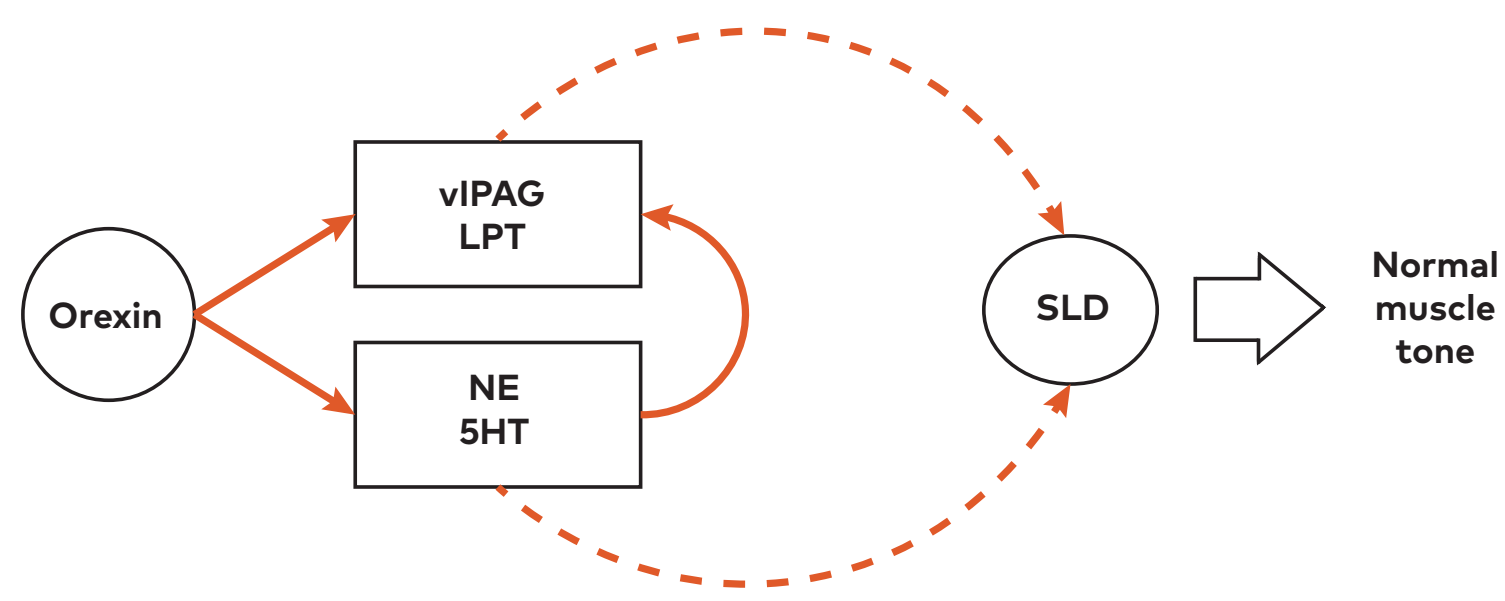

Figure 1. Suggested pathway for normal muscle tone. During normal wake periods in noncataleptic individuals, orexin stimulation of the vIPAG/LPT nuclei and monoaminergic centers, leads to adequate inhibition of the sublaterodorsal nuclei and hence, maintenance of normal muscle tone. Adapted with permission from Burgess et al..$^{18}$ vIPAG: ventrolateral periaqueductal grey, LPT: lateral pedunculopontine tegmentum, NE: norepinephrine, 5HT: 5-hydroxytryptamine (Serotonin), SLD: sublaterodorsal nuclei. Solid line: stimulation; Dashed line: inhibition; line thickness correlates with strength

peptides synthesized in the lateral hypothalamus that have wide excitatory projections to the nuclei in the arousal system..$^{-11,13-15}$ The orexins promote wakefulness with innervation and excitation of key nuclei in both the dorsal and ventral aspects of the arousal system. ${ }^{13,19}$ They also play a stabilizing role, maintaining consolidated sleep and wakeful periods. Patients suffering from narcolepsy with cataplexy have been shown to be deficient in orexin signaling, with loss of almost $90 \%$ of their orexin-producing neurons. ${ }^{13,18}$

Cataplexy is thought to be the result of intrusion of incomplete REM into wakefulness. Activation of the same brain regions occur in both REM sleep atonia and cataplexy. ${ }^{18} \mathrm{~A}$ comprehensive review of the complex circuitry involved in sleep regulation is beyond the scope of this report. Salient points are discussed below.

The sublaterodorsal nucleus (SLD), a group of neurons in the caudal pons, plays a significant role in REM sleep., ${ }^{4,11}$ Glutamatergic neurons in the SLD project to the ventromedial medulla, stimulating GABAergic and glycinergic interneurons, resulting in the atonia seen during REM sleep. For purposes of this discussion, the SLD can be considered a gatekeeper to muscle atonia observed in REM sleep and cataplectic attacks.
The SLD receives inhibitory inputs from monoaminergic neurons and, the ventrolateral periaqueductal grey matter ( $V I P A G$ ) and the lateral pedunculopontine tegmentum (LPT), and excitatory inputs from the central nucleus of the amygdala. ${ }^{4,11,13,14,16,18,19}$ The amygdala, a key region for processing emotional stimuli, also sends inhibitory projections to the vIPAG/ LPT., 518 During normal wake periods, the vIPAG/ LPT send inhibitory projections to the SLD, thereby opposing SLD mediated muscle atonia. ${ }^{18}$ (Figure 1) However, in cataplexy, unopposed projections from the amygdala to the SLD reduce muscle tone when processing positive emotional stimuli, resulting in muscle atonia and postural failure. In healthy individuals, the propensity of these projections to cause cataplectic atonia is prevented by an increase in the hormone orexin during positive emotions, as well as the monoaminergic and GABAergic vIPAG/LPT inhibition of the SLD

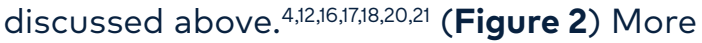
broad arousal system networks not known to be involved in cataplexy include activating projections to the cortex, involving neuronal pathways from the brainstem reticular formation, thalamus, posterior hypothalamus, and basal forebrain..$^{19}$

Treatment of traditional cataplexy has centered on the use of antidepressants. The first antidepressants reported as efficacious were tricyclic 


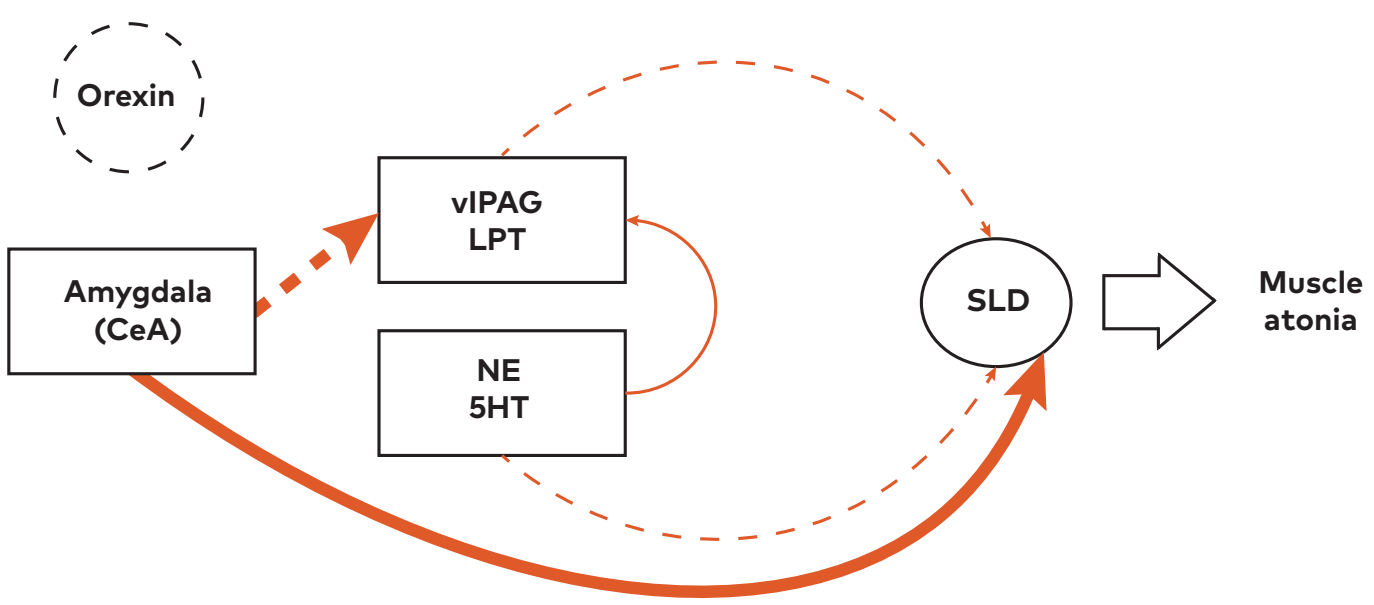

Figure 2. Suggested pathway for muscle atonia. In cataplectic patients, it is hypothesized that processing of positive (and some negative) stimuli by the CeA, inhibits the VIPAG/LPT and stimulates the SLD, overcoming the vIPAG/LPT and monoaminergic inhibition. This leads to REM-type atonia during wakefulness and symptoms of cataplexy. Adapted with permission from Burgess et al. ${ }^{18}$ vIPAG: ventrolateral periaqueductal grey, LPT: lateral pedunculopontine tegmentum, NE: norepinephrine, 5HT: 5-hydroxytryptamine (Serotonin), SLD: sublaterodorsal nuclei, CeA: contral nucleus of the amygdala. Solid line: stimulation; Dashed line: inhibition; line thickness correlates with strength

antidepressants. ${ }^{4}$ The most commonly used tricyclic antidepressants for cataplexy have been imipramine, protryptiline, and clomipramine. Selective serotonin reuptake inhibitors have been also been used, but relatively high doses are required which results in greater adverse effects. ${ }^{5}$ Low to moderate doses of SNRI antidepressants may be of most benefit with the lowest side effect profiles, although there is a lack of controlled evidence., ${ }^{3,4}$ Sodium oxybate is another drug that has shown efficacy in treating cataplexy and is approved by the Food and Drug Administration and the European Medicines Agency for this indication. ${ }^{5}$ Sodium oxybate is the sodium salt of $\beta$-hydroxybutyrate. Its exact mechanism of efficacy is unknown but may involve GABA-B receptors, causing an activation of slow-wave sleep..$^{5}$ The drug unfortunately is expensive, making SNRI antidepressants still the best option for most individuals.

This case is believed to be the first reported of perampanel-induced cataplexy. The pathophysiology is unknown but likely involves the glutamate pathway. It is unlikely that the cataplexy seen in our patient resulted from perampanel-inhibiting, orexin-secreting neurons of the lateral hypothalamus or action as an orexin receptor antagonist, as the reduction of diffuse orexin innervation of the arousal and sleep-in- ducing systems would result in some form of sleep disorder such as narcolepsy, hypnagogic or hypnopompic hallucinations, sleep paralysis or REM sleep behavior disorder. Our patient had no reported or observed complaints of sleep disorder.

We posit that in our patient, perampanel was reducing the wakeful inhibition of the SLD, allowing the amygdala to induce atonia. If perampanel works only by inhibiting AMPA receptors in the brain, ${ }^{3}$ then a possible mechanism of cataplexy in our patient could be an inhibition of the VIPAG/LPT GABAergic or LC/DR monoaminergic projections to the SLD. Given the absence of any sleep disorder, the latter is less likely and a perampanel-vIPAG/LPT interaction is more likely.

\section{Conclusion}

Perampanel-induced cataplexy should be included in the differential diagnosis of perampanel-treated patients presenting with lability concerns. As the use of perampanel increases, more neurologists, primary care providers, hospitalists and psychiatrists may see more epileptic patients with perampanel-related psychiatric-domain adverse effects, such as irritability or aggression, as well as memory loss, as in our patient. Increased awareness of cataplexy and other psychiatric presentations as adverse 
effects of perampanel will lessen the possibility that those affected could be inappropriately treated with antipsychotics or benzodiazepines for what may be mistaken for a mood or other organic mental disorder.

\section{Acronyms}

DR: dorsal and median raphe nuclei (monoaminergic-5-HT)

LC: locus coeruleus (monoaminergic-NE)

TMN: Tuberomamillary nuclei (monoaminergic-HA)

LPT: Lateral pontine tegmentum

vIPAG: ventrolateral Periaqueductal gray matter

\section{Conflicts of Interest}

Dr. Gracious reports personal fees from Novo Nordisc not related to the submitted work.

Drs. Bond, Gracious and Zafar are employees of Orange Park Medical Center, a hospital affiliated with the journal's publisher.

This research was supported (in whole or in part) by HCA Healthcare and/or an HCA Healthcare affiliated entity. The views expressed in this publication represent those of the author(s) and do not necessarily represent the official views of HCA Healthcare or any of its affiliated entities.

\section{Author Affiliation}

1. Edward Via College of Osteopathic Medicine, Auburn, AL

2. Orange Park Medical Center, Orange Park, FL

\section{References}

1. Greenwood J, Valdes J. Perampanel (Fycompa): A Review of Clinical Efficacy and Safety in Epilepsy. P T. 2016;41(11):683-698. https://www.ncbi. nlm.nih.gov/pmc/articles/PMC5083075/

2. Fycompa [package insert]. Woodcliff Lake, NJ: Eisai Inc.; 2016.

3. Faulkner MA. Spotlight on perampanel in the management of seizures: design, development and an update on place in therapy. Drug Des Devel Ther. 2017;11:2921-2930. Published 2017 Oct 4. https://doi.org/10.2147/dddt.s122404

4. Dauvilliers Y, Siegel JM, Lopez R, Torontali ZA, Peever JH. Cataplexy--clinical aspects, pathophysiology and management strategy. Nat Rev Neurol. 2014;10(7):386-395. https://doi. org/10.1038/nrneurol.2014.97

5. Pillen S, Pizza F, Dhondt K, Scammell TE, Overeem S. Cataplexy and Its Mimics: Clinical Recognition and Management. Curr Treat Options Neurol. 2017;19(6):23. https://doi.org/10.1007/ s11940-017-0459-0

6. Egel RT, Lee A, Bump T, Javois A. Isolated cataplexy in the differential diagnosis of drop attacks: a case of successful clinical diagnosis and treatment. Case Rep Neurol Med. 2012;2012:757586. https://doi. org/10.1155/2012/757586

7. Gauci S, Hosking W, Bruck D. Narcolepsy, cataplexy, hypocretin and co-existing other health complaints: A review. Cogent Med. 2017;4(1):1312791. https://doi.org/10.1080/233120 $\underline{\text { 5X.2017.1312791 }}$

8. Alonso-Navarro H, Montes JM, Plaza-Nieto JF, Jiménez-Jiménez FJ. Cataplexy Possibly Associated With Lamotrigine. J Clin Psychopharmacol. 2016;36(4):400-402. https://doi.org/10.1097/ jcp.0000000000000532

9. Saper CB, Scammell TE, Lu J. Hypothalamic regulation of sleep and circadian rhythms. Nature. 2005;437(7063):1257-1263. https://doi. org/10.1038/nature04284

10. España RA, Scammell TE. Sleep neurobiology from a clinical perspective. Sleep. 2011;34(7):845858. Published 2011 Jul 1. https://doi.org/10.5665/ sleep.1112

11. Saper CB. The neurobiology of sleep. Continuum (Minneap Minn). 2013;19(1 Sleep Disorders):19-31. https://doi.org/10.1212/01. con.0000427215.07715.73

12. Liblau RS, Vassalli A, Seifinejad A, Tafti M. Hypocretin (orexin) biology and the pathophysiology of narcolepsy with cataplexy. Lancet Neurol. 2015;14(3):318-328. https://doi.org/10.1016/s14744422(14)70218-2

13. Scammell TE. Narcolepsy. N Engl J Med. 2015;373(27):2654-2662. https://doi.org/10.1056/ nejmra1500587

14. Scammell TE. Overview of sleep: the neurologic processes of the sleep-wake cycle. J Clin Psychiatry. 2015;76(5):e13. https://doi.org/10.4088/ jcp.14046tx1c

15. Scammell TE, Arrigoni E, Lipton JO. Neural Circuitry of Wakefulness and Sleep. Neuron. 2017;93(4):747-765. https://doi.org/10.1016/j.neuron.2017.01.014

16. Mahoney CE, Agostinelli LJ, Brooks JN, Lowell BB, Scammell TE. GABAergic Neurons of the Central Amygdala Promote Cataplexy. J Neurosci. 2017;37(15):3995-4006. https://doi.org/10.1523/ ineurosci.4065-15.2017

17. Blouin AM, Siegel JM. Relation of melanin concentrating hormone levels to sleep, emotion and hypocretin levels. Sleep. 2013;36(12):1777. Published 2013 Dec 1. https://doi.org/10.5665/ sleep.3194 
18. Burgess CR, Scammell TE. Narcolepsy: neural mechanisms of sleepiness and cataplexy. J Neurosci. 2012;32(36):12305-12311. https://doi. org/10.1523/jneurosci.2630-12.2012

19. Jones BE. Arousal systems. Front Biosci. 2003;8:s438-s451. Published 2003 May 1. https:// doi.org/10.2741/1074

20. Taylor NE, Pei J, Zhang J, et al. The Role of Glutamatergic and Dopaminergic Neurons in the Periaqueductal Gray/Dorsal Raphe: Separating Analgesia and Anxiety. eNeuro. 2019;6(1):ENEURO.0018-18.2019. https://doi.org/10.1523/ENEURO.0018-18.2019

21. Krenzer M, Anaclet C, Vetrivelan R, et al. Brainstem and spinal cord circuitry regulating REM sleep and muscle atonia. PLoS One. 2011;6(10):e24998. https://doi.org/10.1371/journal. pone.0024998 